\title{
Passive coherent dual-comb spectroscopy based on optical-optical modulation with free running lasers
}

\author{
Chenglin Gu', Zhong Zuo ${ }^{1}$, Daping Luo ${ }^{1}$, Zejiang Deng ${ }^{1}$, Yang Liu', Minglie Hu² and Wenxue $\mathrm{Li}^{1 *}$ (D)
}

\author{
* Correspondence: wxli@phy.ecnu. \\ edu.cn \\ ${ }^{1}$ State Key Laboratory of Precision \\ Spectroscopy, East China Normal \\ University, Shanghai 200062, China \\ Full list of author information is \\ available at the end of the article
}

\begin{abstract}
Dual-comb spectroscopy is a powerful spectroscopic tool with ultrahigh-resolution, high-sensitivity properties, which opens up opportunities for the parallel detection of multi-species molecules. However, in its conventional form, highly stable laser combs with sophisticated control systems are required to perform dual-comb spectroscopy. Here, a passive mutually coherent dual-comb spectroscopy system via an opticaloptical modulation method is addressed, where all fast phase-locking electronics are retired. Without post computer-based phase-correction, a high degree of mutual coherence between the two combs with a relative comb-tooth linewidth of $10 \mathrm{mHz}$ is achieved, corresponding to a coherent time of $100 \mathrm{~s}$. To demonstrate the performance and versatility of the system, the dual comb spectrometer is applied to record the mode-resolved single molecular spectra as well as parallel detected spectra of mixed gases including $\mathrm{CO}_{2}, \mathrm{CO}$ and $\mathrm{C}_{2} \mathrm{H}_{2}$ that well agree with the established spectral parameters. Our technique exhibits flexible wavelength tuning capability in the near-infrared region and can be potentially extended to the midinfrared region for more applications.
\end{abstract}

Keywords: Optical frequency combs, Comb spectroscopy, Optical-optical modulation, Molecular spectroscopy

\section{Introduction}

Laser frequency comb spectroscopy with enhanced measurement speed, sensitivity, and precision has been used in various applications [1,2], including distance metrology [3], molecular precision spectroscopy [4], chemical catalysis [5], biological analysis [6], environmental monitoring [7], and clinical pathology [8]. Among different laser comb technologies, dual-comb spectroscopy offers the prospect of surpassing the widely used Fourier transform spectroscopy (FTS) techniques. Dual-comb spectroscopy (DCS), which measures the time-domain interference between two combs with slightly different line spacing, is capable of very fast spectral measurements with high precision and sensitivity $[9,10]$. In the frequency domain, the technique can be understood as a multi-heterodyne detection of pairs of neighboring comb lines, by which the optical frequency combs are down-converted to a single radio-frequency comb [11]. However, the technique of dual-comb spectroscopy has not yet realized its full potential, mostly because of the difficulty of synchronizing the pulse trains of two comb lasers to achieve

(c) The Author(s). 2020 Open Access This article is distributed under the terms of the Creative Commons Attribution 4.0 International License (http://creativecommons.org/licenses/by/4.0/), which permits unrestricted use, distribution, and reproduction in any medium, provided you give appropriate credit to the original author(s) and the source, provide a link to the Creative Commons license, and indicate if changes were made. 
a narrow relative linewidth between the pairs of teeth of the two combs. In other words, the timing and phase between the two pulses must be accurate to within a fraction of an optical cycle $[12,13]$.

Most commonly used sources for optical dual-comb are based on two mutual coherent mode-locked lasers. The coherence of these dual-comb sources is typically ensured by phase-locking both combs together to the same pair of cavity-stabilized continuous-wave lasers with several forward or backward fast feedback loop system, which make the instrumentation design of dual-comb systems complex and expensive [14-16]. Furthermore, it makes operating the dual-comb system outside a well-maintained metrology laboratory practically impossible. The other approach corrects the phase fluctuation and timing jitter via real-time compensation electronics [17, 18], or in a posterior process [19], by deriving adaptive phase correction signals from two continuous-wave $(\mathrm{CW})$ lasers, even with free running lasers. The mutual coherence time of approximately $1 \mathrm{~s}$ is a strong limitation of the techniques due to electronic instrument noise introduced in the phase detection or locking process $[20,21]$. To reduce the cumbersome control electronics design systems with a built-in passive mutual coherence is a promising current trend [22, 23]. Another emerging comb source, the electro-optic modulator (EOM) comb, operates by combining the intensity modulations of a single CW laser with two EOMs to generate mutually coherent dual combs $[24,25]$. To transmit dual-comb spectroscopy out of the lab and into practical use, the stabilization of system should be simplified but the quality and speed of the combs should be still stained. To achieve the potential of dual-comb spectroscopy with high stability and coherence, new concepts and approaches must be devised.

Here, we demonstrated an optical method to develop an inherent passive mutually coherent DCS source, where mode-resolved spectra can be obtained without any fast electronics. The technique, called optical-optical modulated frequency combs (OMFCs), only requires free running femtosecond oscillators with repetition rates referenced to a radio-frequency (RF) clock. Significant improvements in robustness and stability than conventional dualcomb sources are obtained. In the experiment, a high degree of mutual coherence between the two OMFCs with a relative comb-tooth linewidth down to $10 \mathrm{mHz}$ (the corresponding mutual coherence time is $100 \mathrm{~s}$ ) is demonstrated. First, we describe the principle of OMFC dual-comb spectroscopy. Then we show its experimental performance with broad highresolution molecular absorption features. We finally summarize this paper and discuss the new opportunities opened up by OFMC dual-comb spectroscopy.

\section{Methods and experimental}

Frequency combs must be actively stabilized or monitored to remove noise in both the carrier frequency and repetition rate [26]. In contrast with combs usually actively stabilized against continuous-wave lasers, we generate combs by directly pulsing the $\mathrm{CW}$ laser using an ultrafast optical modulation technique. The OMFCs are realized via a $\mathrm{CW}$-seeded optical parametric amplification (OPA) process. The seeded signal laser, a narrow linewidth $\mathrm{CW}$ laser, is pulsed and amplified by a free-running femtosecond pump laser, which here serves as a broad bandwidth optical modulator. The superior gain and noise dynamics of $\mathrm{CW}$ injection seeded OPA with remarkable pulse-to-pulse and long-term power stability are already demonstrated [27, 28]. The pulsed signal maintains excellent coherence with the input CW signal laser in the pure optical second nonlinear parametric process [29]. In the time domain, the carrier frequency of 
the signal is exact at the frequency of the injection CW seed. It is to say that one tooth of the signal comb is inherited from the frequency line of the CW laser in the frequency domain. Therefore, the pulse signal is passively locked to the CW laser without any active beat detection or locking process. Additionally, the generated frequency combs have also been amplified to a considerable power simultaneously and maintain a fine pulse quality in the time domain. The fine pulse quality will be preferred in some nonlinear DCS applications $[6,30]$. In another dimension, the repetition rates of OFMCs are equal to the repetition rates of the femtosecond pump lasers which are coarsely referenced to a Rubidium frequency standard via the cavity mirror mounted on a piezoelectric transducer with a relatively low bandwidth of $30 \mathrm{~Hz}$.

The experimental setup is presented in Fig. 1. Two mode-locked ytterbium-doped fiber laser amplifiers are emitting pulses at slightly different repetition frequencies $\left(f_{\text {rep} 1 \text {, }}\right.$ $2 \approx 108 \mathrm{MHz}$ ) and $\lambda=1040 \mathrm{~nm}$. The output of a CW laser diode, with an optical frequency $f_{\mathrm{cw}}$, is divided into two arms. Each CW laser is pulsed and amplified by an OPA process pumped with the ytterbium-doped femtosecond fiber laser in a temperaturecontrolled 10-mm-long MgO-doped periodically poled lithium niobate (PPLN) crystal. The average power of each comb exceeds $500 \mathrm{~mW}$ when the power of the pump is $3 \mathrm{~W}$ and that of the CW seed $\sim 5 \mathrm{~mW}$. The quantum conversion efficiency is approximately $25 \%$, which can be expected to increase when a longer nonlinear crystal or double pass configuration is used. After the PPLN crystals, optical long-wavelength pass filters filter out the light of the pump and of the idler. Two mutually coherent dual combs are generated with a carrier frequency of $f_{\mathrm{cw}}$ and line spacing of $f_{\text {rep1 }}$ and $f_{\text {rep } 2}=f_{\text {rep } 1}+\Delta$, where $\Delta$ is the difference of the repetition rate of the pump sources. The mutual coherence between the two combs is established by sharing the same seeded CW frequency and from the inherent mechanism of the OPA process.

The loose reference with low feedback bandwidth can suppress the long-term drifts of repetition rates of OFMCs, and the residual short-term instability limit the coherence of OFMCs within a relative narrow bandwidth due to the cumulative effect. For bandwidth within $\sim 3 \mathrm{~nm}(0.4 \mathrm{THz})$, well tooth resolved DCS with tooth bandwidth $2 \mathrm{~Hz}$ is obtained ( $0.5 \mathrm{~s}$ coherent time) (see Additional file 1 for detailed). For broadband DCS where short-term stability of repetition rate is required, adaptive sampling method is adopted to cancel the distortion as illustrated in Fig. 2. It is worth mentioning that dual-comb spectroscopy based on OMFCs with highly mutual coherence has a great advantage over free running lasers as comb sources when adopting the adaptive

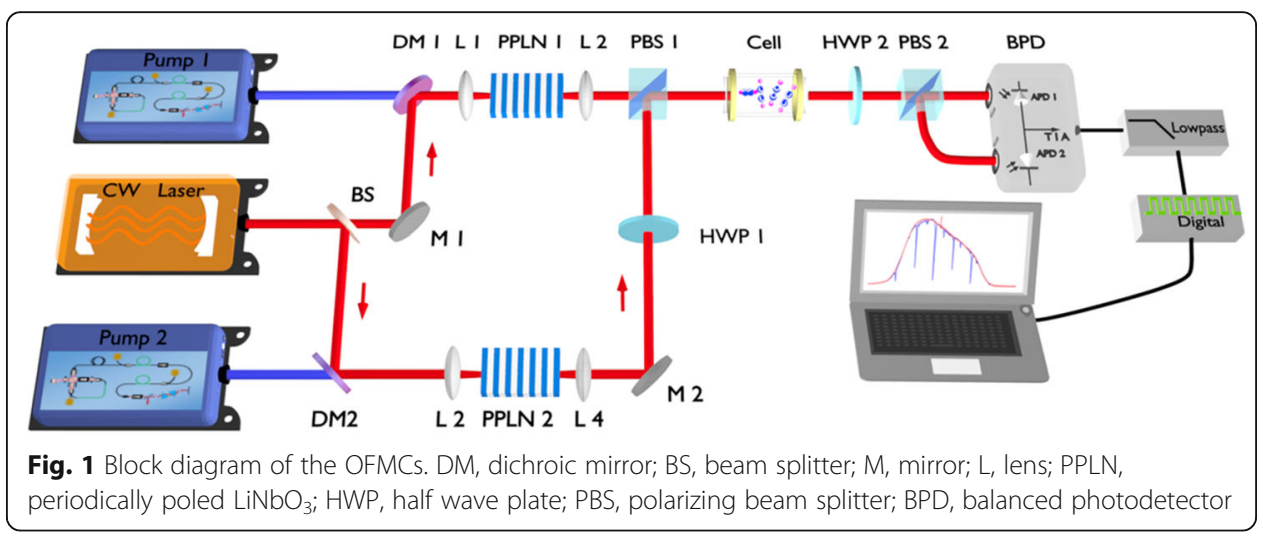




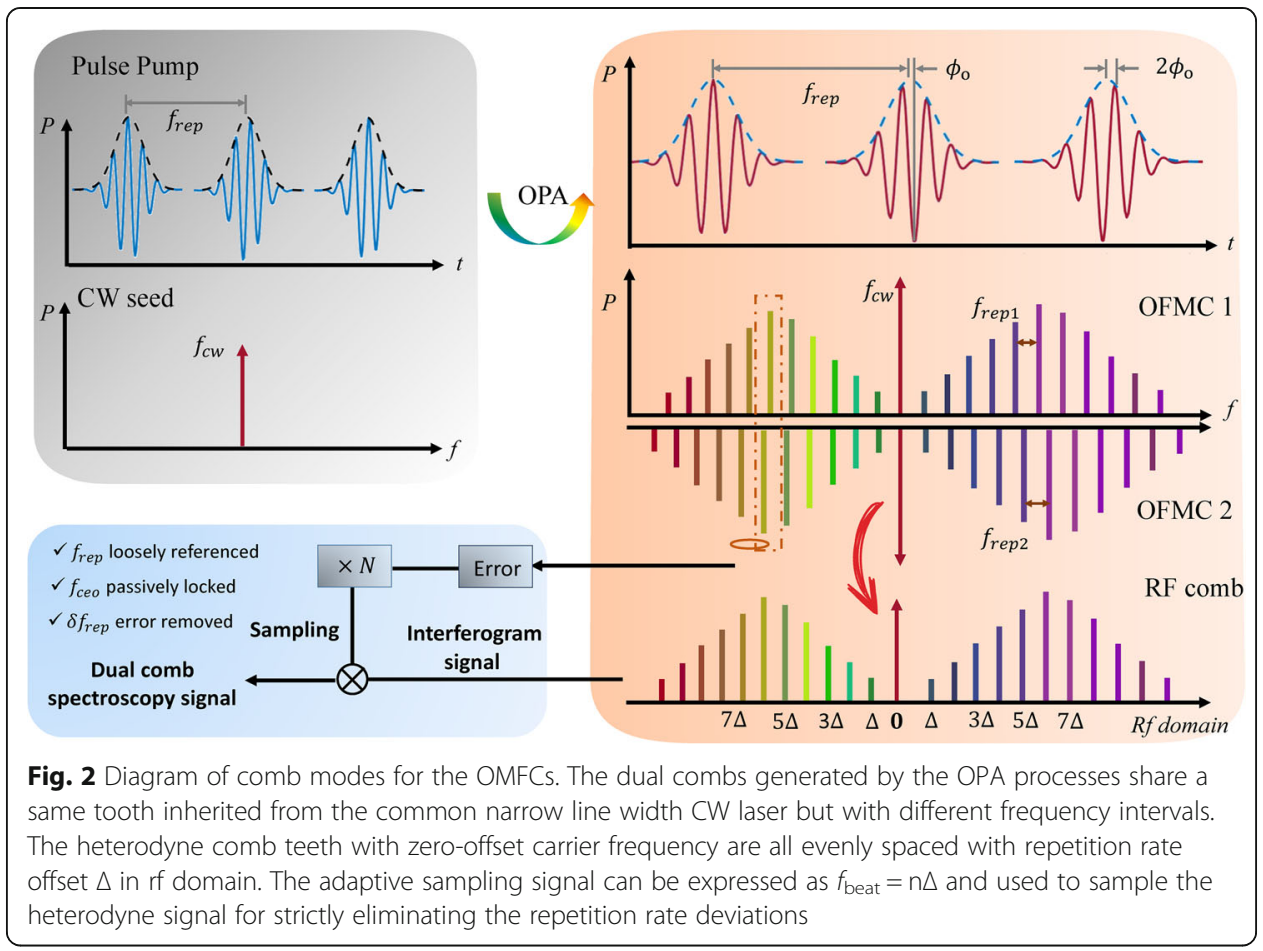

sampling method. The OFMCs inherit the common CW frequency and only the fluctuations of one dimension, the repetition rates, need to be removed. Compared to the free-running lasers, the adaptive sampling technique used here only need another $\mathrm{CW}$ laser as the frequency intermediate. The loose reference of repetition rate in our experiment is mainly used to maintain the electrical signals not beyond the half of repetition rate and achieve long time running, where the relative frequency shift between $\mathrm{CW}$ lasers occupies the dominate impact. In free-running lasers, the phase variations of the carrier-envelope offset drive the comb tooth drift in a wide range in the RF spectrum; and, so is the beat signal. At the time when the beat signal is too close, even aliases with the repetition rate, or drifts out of the bandwidth of the filter, the filter will fail to filter out the beat signal. These cause the stability time of DCS with free-running laser to reach its limit at ten of seconds and our system can be continuously operation for weeks without any adjustment [17].

\section{Results and discussion}

The performance of our passive mutual coherent DCS is characterized. The beams from the two OMFCs with average power $\sim 5 \mathrm{~mW}$ in each beam are spatially combined and then pass through a multipass gas cell. The high mutual coherent pulse trains periodically and repeatedly move through each other, resulting in stable interferograms at an offset of repetition frequencies $\Delta f=300 \mathrm{~Hz}$. No post processing (except for fast Fourier transform (FFT)) of the raw data was performed in our experiments. A typical detector signal is shown in Fig. 3a, and the enlarged interferograms at $1 \mathrm{~s}, 50 \mathrm{~s}$ and 100 $\mathrm{s}$ show the stability of the phase of the signal that is beneficial for coherent averaging. The resulting retrieved DCS spectra are shown in Fig. 3b, and we did not apply apodization when performing FFT. The tooth-resolved spectra, covering $\sim 55 \mathrm{~nm}(\sim 48,000$ 


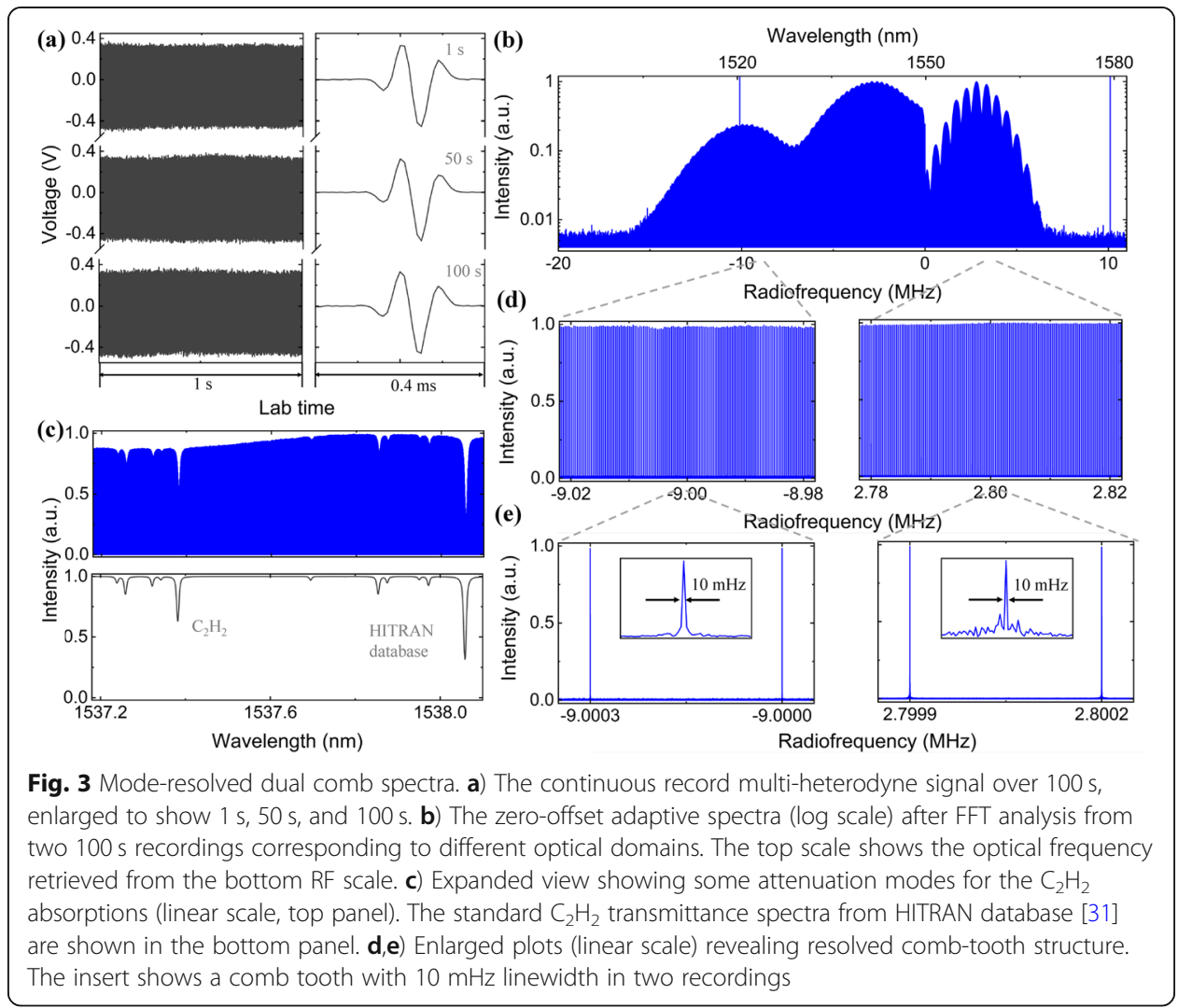

teeth), are obtained by two independent measurements with filters centered at different wavelengths each from a single $100 \mathrm{~s}$ long recording time. The full width at half maximum (FWHM) RF linewidth of the individual comb lines is $10 \mathrm{mHz}$ (limited by the Fourier transform time) in the radiofrequency spectrum (3.3 kHz in the optical domain) as shown in Fig. 3e. The limitation of the coherent time in our experiment is mainly due to the noise introduced by the electronic circuit for adaptive signal detection. This noise causes residual fluctuation of the difference of the repetition frequencies resulting in sidebands of the tooth as shown in Fig. 3e. Figure 3c shows the typical comb-toothresolved optical absorption spectrum of acetylene $\left(\mathrm{C}_{2} \mathrm{H}_{2}\right)$, which shows good consistency with the theoretical transmission spectrum from the HITRAN database.

We illustrate the potential of our system for molecular spectroscopy with the absorption lines in the region of the $\mathrm{P}$ branch of $\mathrm{C}_{2} \mathrm{H}_{2}$. We first adjust the center wavelength of the combs to cover the absorption spectrum domain of $\mathrm{C}_{2} \mathrm{H}_{2}$ by adjusting the grating period of the PPLN. A multi-pass gas cell of length $\mathrm{L}=8 \mathrm{~cm}$ is filled with 29 mbar $\mathrm{C}_{2} \mathrm{H}_{2}$ of natural abundance $\left(\sim 2 \% \mathrm{H}^{12} \mathrm{C}^{13} \mathrm{CH}\right.$ and $\left.\sim 98 \% \mathrm{H}^{12} \mathrm{C}^{12} \mathrm{CH}\right)$. The wavelength calibration is derived from the measurement of the $\mathrm{CW}$ laser wavelengths and the comb line spacing, which equals to the repetition rate of the pump laser. The absorption lines of $\mathrm{C}_{2} \mathrm{H}_{2}$ are shown in Fig. 4a, obtained by coherently averaging 150,000 interferograms in the time domain, corresponding to $500 \mathrm{~s}$ recording time. Phase correction is performed every $100 \mathrm{~s}$ recording our mutual coherent time. Portions of the absorption lines are shown in Fig. $4 \mathrm{~b}$, and the fine modulation structure on the spectrum is clearly visible. The experimental profiles of ro-vibrational lines (Fig. 4c) are compared to the computed spectra from the HITRAN database. We enlarge some rotational- 

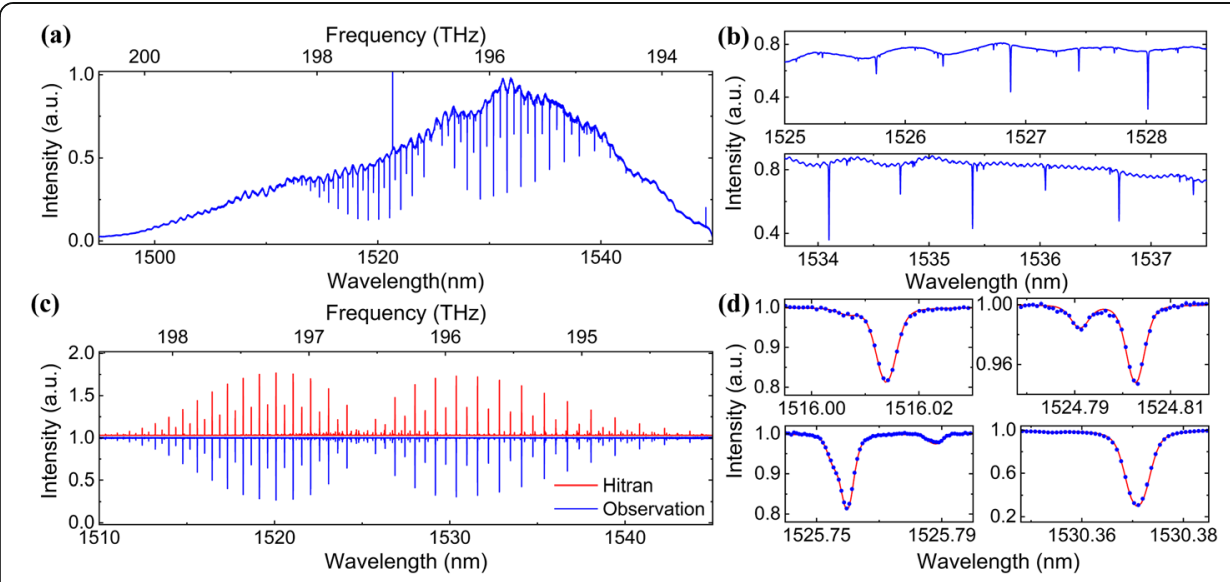

Fig. 4 Measured spectrum of $\mathrm{C}_{2} \mathrm{H}_{2}$. a) The spectrum obtained from a continuous $500 \mathrm{~s}$ recording, phase corrected every $100 \mathrm{~s}$ and averaged. Absorption lines originate from the 8-cm-long gas cell filled with 29 mbar $\mathrm{C}_{2} \mathrm{H}_{2}$ of natural abundance. b) Portions of the absorption lines in (a), which show fine modulation structure clearly in the bottom panel. c) Comparison between the extracted absorption lines (blue line) and the theoretical absorbance spectra (red line) of $\mathrm{C}_{2} \mathrm{H}_{2}$ computed using the HITRAN line parameters. d) Portions of absorption lines in (c), showed good agreement between the measured absorption lines (blue scatter) and the curve profiles from HITRAN

vibrational lines at different wavelengths and compare them with the lines of the standard database, as shown in Fig. 4d. The widths of these Doppler-broadened absorption lines are approximately $600 \mathrm{MHz}$, which is $\sim 200 \mathrm{MHz}$ broader than Doppler lines. A week absorption line down to $2 \%$ is clearly visible. The residuals between the experimental and calculated spectra have a standard deviation of approximately $0.2 \%$ at the noise level.

The spectrum of the comb signal is broadened to $1500-1620 \mathrm{~nm}$ in highly nonlinear fiber (HNLF) to demonstrate parallel spectroscopic detection of multiple gases. The gas cell is a $4.8 \mathrm{~m}$ optical path and filled with about $450 \mathrm{mbar} \mathrm{CO}, 200 \mathrm{mbar} \mathrm{CO}_{2}$ and 1.8 mbar $\mathrm{C}_{2} \mathrm{H}_{2}$. Two independent measurements, each with wavelength covering from $1500 \mathrm{~nm}$ to $1550 \mathrm{~nm}$ and $1550 \mathrm{~nm}$ to $1620 \mathrm{~nm}$, are applied to avoid arising in the RF frequency domain. We coherently average 15,000 interferograms with $50 \mathrm{~s}$ recording time. For the DCS with supercontinuum from HNLF, the presence of a noise seed on the input laser pulse can lead to significant excess noise on the generated output supercontinuum electric field [21]. This noise degrades the mutual coherence of the supercontinuum combs, and the limitation of the mode tooth in the RF domain is $0.2 \mathrm{~Hz}$ corresponding to $5 \mathrm{~s}$ coherent time. The mutual coherence could be improved by using a single nonlinear fiber, in which the two pulse trains counterpropagate for efficient common-noise rejection and to maintain the mutual coherence between the two combs [25]. The retrieved DCS spectra in Fig. 5a shows the characteristic absorption features of the different molecules. We compare the absorption lines with a spectrum computed from the HITRAN database as an illustration of the excellent quality of our experimental spectra (Fig. 5b). The signal-to-noise ratio is at best 378 at approximately $192 \mathrm{THz}$ and the average signal-to-noise ratio is 183 over $185-200 \mathrm{THz}$, which leads to a figure of merit of $3.9 \times 106 \mathrm{~Hz}^{1 / 2}$. Some rotational-vibrational lines from different molecules together with lines from a standard database are enlarged as shown in Fig. 5c. 

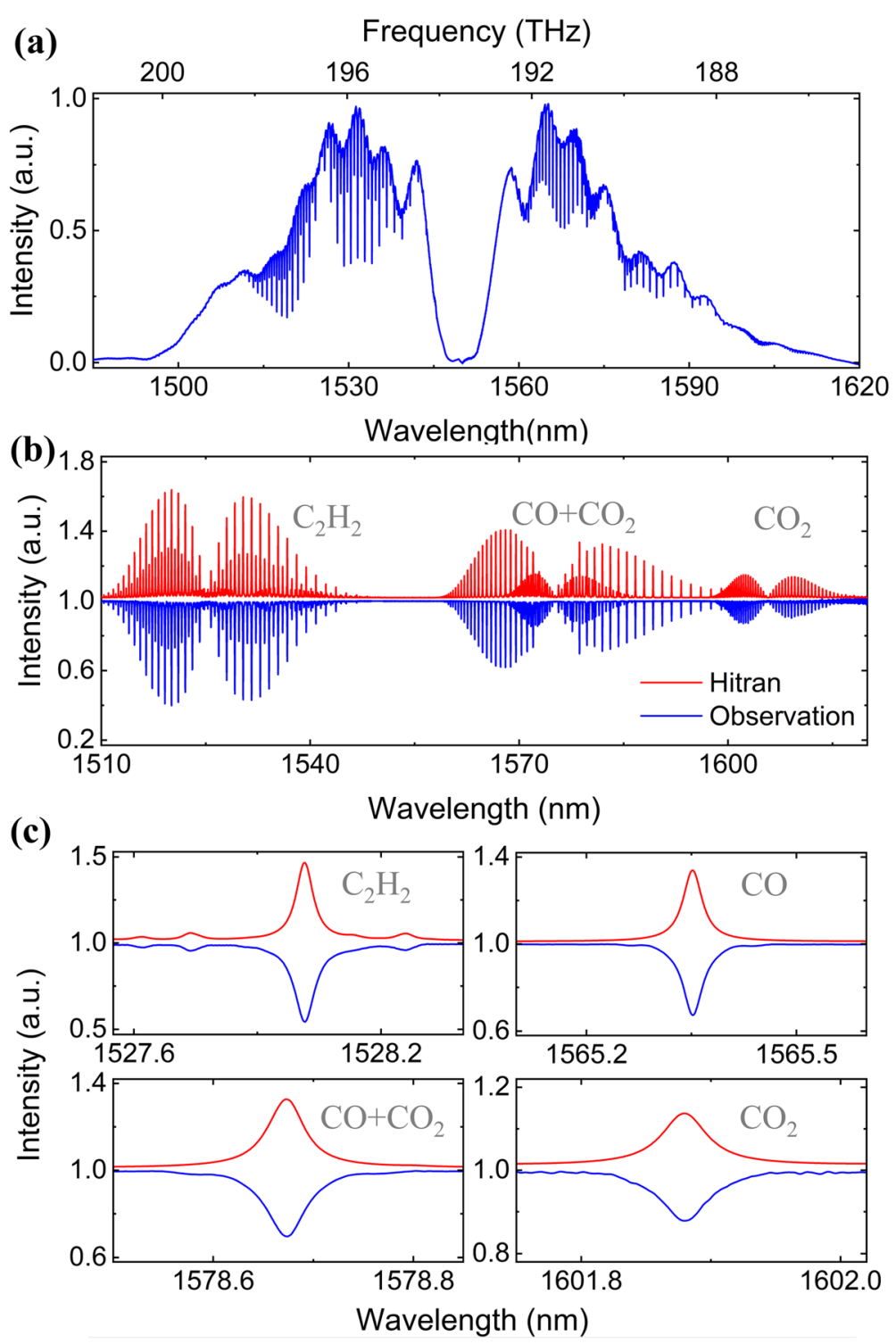

Fig. 5 Dual comb spectra of the gas mixture. a) The spectra obtained from two independent measurements, recording $50 \mathrm{~s}$ and averaged. The gas cell is 4.8-m-long and filled with approximately 450 mbar CO, 200 mbar $\mathrm{CO}_{2}$ and $1.8 \mathrm{mbar}_{2} \mathrm{H}_{2}$. b) Comparison between the extracted absorption lines (blue line) and the theoretical result (red line) computed based on HITRAN database. c) Portions of the absorption lines for different gases. The measurement absorption intensity agrees well with the theoretical (red lines)

\section{Conclusions}

We first proposed an optical modulated comb and demonstrated its use in dual comb spectroscopy. High mutual coherence of the dual comb generated by the OPA process makes it possible to resolve comb lines of a Fourier-limited width of approximately 10 $\mathrm{mHz}$ without active carrier envelop phase stabilization at high signal-to-noise ratio (SNR). Note that the method can be easily adopted in the mid-infrared region with a CW mid-infrared laser and can be extended into the far-infrared region with the development of nonlinear crystal technology, and we already have some initial experiment results at 3 4 um with the similar experimental configure [32]. With further system 
development, a wider spectrum and better SNR can be achieved, and with the aid of nonlinear crystal waveguide, the power required for the pump laser would dramatically decrease. The fine pulse quality of the generated pulse makes it possible for use in nonlinear optics applications. Our technique offers a simple and robust approach to generate optical dual-comb sources and is of great significance for the popularization of the applications of dual-comb spectroscopy.

\section{Supplementary information}

Supplementary information accompanies this paper at https://doi.org/10.1186/s43074-020-0005-2.

Additional file 1: Figure S1. Narrow bandwidth dual OFMCs. a) The mode-resolved spectra from a $0.5 \mathrm{~s}$ recording time. b) Expanded view showing the individual teeth in different positions of (a), respectively. Figure S2. Dual comb absorption spectroscopy of $\mathrm{C}_{2} \mathrm{H}_{2}$. a) The averaged spectra before and after the gas cell. b) Comparison between the extracted absorption lines and the theoretical computed result. Figure S3. Serial spectra of the OFMC obtained by scanning the CW wavelength. Figure S4. The measured electrical signal of adaptive signal, the beats between the OFMCs and intermediate CW laser. The insert shows the zoomed result of $f_{s}$ with a 3-dB bandwidth of $20 \mathrm{kHz}$ (RBW: $10 \mathrm{kHz}$ ).

\section{Abbreviations}

FTS: Fourier transform spectroscopy; DCS: Dual comb spectroscopy; EOM: Electro-optic modulator; OMFC: Opticaloptical modulated frequency comb; CW: Continuous-wave; RF: Radio-frequency; OPA: Optical parametric amplification; PPLN: Periodically poled lithium niobate; FFT: Fast Fourier transform; FWHM: Full width at half maximum; HNLF: Highly nonlinear fiber; SNR: Signal-to-noise ratio

\section{Acknowledgements}

We thank Professor Ming Yan from East China Normal University for effective discussions.

\section{Authors' contributions}

CG and ZZ contributed equally to this work. WL initiated and supervised the project. All authors contributed extensively to the work presented in this paper. All authors read and approved the final manuscript.

\section{Funding}

National Key R\&D Program of China (2017YFF0206000, 2018YFA0306301); National Natural Science Foundation of China (11904105, 11874153, 11804096).

\section{Availability of data and materials}

The datasets used and/or analyzed during the current study are available from the corresponding author on reasonable request.

\section{Competing interests}

The authors declare that they have no competing interests.

\section{Author details}

${ }^{1}$ State Key Laboratory of Precision Spectroscopy, East China Normal University, Shanghai 200062, China. ${ }^{2}$ Key Laboratory of Optoelectronic Information Science and Technology of Ministry of Education, Tianjin University, Tianjin 300072, China.

Received: 13 November 2019 Accepted: 19 December 2019

Published online: 06 March 2020

\section{References}

1. Udem T, Holzwarth R, Hänsch TW. Optical frequency metrology. Nature. 2002;416:233-7.

2. Mandon J, Guelachvili G, Picqué N. Fourier transform spectroscopy with a laser frequency comb. Nat Photon. 2009;3:99-102.

3. Yokoyama S, Yokoyama T, Hagihara $Y$, Araki T, Yasui T. A distance meter using a terahertz intermode beat in an optical frequency comb. Opt. Express. 2009;17:17324-37.

4. Diddams SA, Hollberg L, Mbele V. Molecular fingerprinting with the resolved modes of a femtosecond laser frequency comb. Nature. 2007;445:627-30.

5. Boudreau S, Levasseur S, Perilla C, Roy S, Genest J. Chemical detection with hyperspectral lidar using dual frequency combs, opt. Express. 2013;21:7411-8

6. Ideguchi T, Holzner S, Bernhardt B, Guelachvili G, Picqué N, Hänsch TW. Coherent Raman spectro-imaging with laser frequency combs. Nature. 2013:502:355-8.

7. Rieker GB, Giorgetta FR, Swann WC, Kofler J, Zolot AM, Sinclair LC, Tans PP. Frequency-comb-based remote sensing of greenhouse gases over kilometer air paths. Optica. 2014;1:290-8.

8. Thorpe MJ, Balslev-Clausen D, Kirchner MS, Ye J. Human breath analysis via cavity enhanced optical frequency comb spectroscopy. Opt. Express. 2008;16:2387-97.

9. Coddington I, Newbury N, Swann W. Dual-comb spectroscopy. Optica. 2016;3:414-26. 
10. Ideguchi T. Dual-Comb Spectroscopy. Opt Photon News. 2017;28:32-9.

11. Schiller S. Spectrometry with frequency combs. Opt Lett. 2002;27:766-8.

12. Okubo S, Iwakuni K, Inaba H, Hosaka K, Onae A, Sasada H, Hong FL. Ultra-broadband dual-comb spectroscopy across 1. 0-1.9 um. Appl Phys Express. 2015;8:082402.

13. Zolot AM, Giorgetta FR, Baumann E, Nicholson JW, Swann WC, Coddington I, Newbury NR. Direct-comb molecular spectroscopy with accurate, resolved comb teeth over 43 THz. Opt Lett. 2012;37:638-40.

14. Coddington I, Swann WC, Newbury NR. Coherent multiheterodyne spectroscopy using stabilized optical frequency combs. Phys Rev Lett. 2008;100:013902.

15. Coddington I, Swann WC, Newbury NR. Coherent dual-comb spectroscopy at high signal-to-noise ratio. Phys Rev A. 2010;82:043817.

16. Chen Z, Yan M, Hänsch TW, Picqué N. A phase-stable dual-comb interferometer. Nat Commun. 2018;9:3035.

17. Ideguchi T, Poisson A, Guelachvili G, Picqué N, Hänsch TW. Adaptive real-time dual-comb spectroscopy. Nat Commun. 2014:5:3375.

18. Roy J, Deschênes JD, Potvin S, Genest J. Continuous real-time correction and averaging for frequency comb interferometry. Opt Express. 2012;20:21932-9.

19. Giaccari P, Deschênes JD, Saucier P, Genest J, Tremblay P. Active Fourier-transform spectroscopy combining the direct RF beating of two fiber-based mode-locked lasers with a novel referencing method. Opt Express. 2008;16:4347-65.

20. Newbury NR, Swann WC. Low-noise fiber-laser frequency combs. J Opt Soc Am B. 2007;24:1756-70.

21. Washburn BR, Newbury NR. Phase, timing, and amplitude noise on supercontinua generated in microstructure fiber. Opt Express. 2004;12:2166-75.

22. Zhao X, Hu G, Zhao B, Li C, Pan Y, Liu Y, Yasui T, Zheng Z. Picometer-resolution dual-comb spectroscopy with a freerunning fiber laser. Opt Express. 2016;24:21833-45.

23. Ideguchi T, Nakamura T, Kobayashi Y, Goda K. Kerr-lens mode-locked bidirectional dual-comb ring laser for broadband dual-comb spectroscopy. Optica. 2016;3:748-53.

24. Long DA, Fleisher AJ, Douglass KO, Maxwell SE, Bielska K, Hodges JT, Plusquellic DF. Multiheterodyne spectroscopy with optical frequency combs generated from a continuous-wave laser. Opt Lett. 2014;39:2688-90.

25. Millot G, Pitois S, Yan M, Hovhannisyan T, Bendahmane A, Hänsch TW, Picqué N. Frequency-agile dual-comb spectroscopy. Nat Photon. 2016:10:27-30.

26. Morohashi I, Sakamoto T, Sotobayashi H, Kawanishi T, Hosako I, Tsuchiya M. Widely repetition-tunable 200 fs pulse source using a Mach-Zehnder-modulator-based flat comb generator and dispersion-flattened dispersion-decreasing fiber. Opt Lett. 2008;33:1192-4.

27. Manzoni C, Cirmi G, Brida D, Silvestri SD, Cerullo G. Optical-parametric-generation process driven by femtosecond pulses: timing and carrier-envelope phase properties. Phys Rev A. 2009;79:033818.

28. Linnenbank H, Steinle T, Giessen H. Narrowband cw injection seeded high power femtosecond double-pass optical parametric generator at 43 MHz: gain and noise dynamics. Opt. Express. 2016;24:19558-66.

29. Chen W, Fan J, Ge A, Song H, Song Y, Liu B, Hu M. Intensity and temporal noise characteristics in femtosecond optical parametric amplifiers. Opt Express. 2017:25:31263-72.

30. Lomsadze B, Smith BC, Cundiff ST. Tri-comb spectroscopy. Nat Photon. 2018;12:676-80.

31. Rothman LS, Gordon IE, Babikov Y, Barbe A, Chris Benner D, Bernath PF, Birk M, Bizzocchi L, Boudon V, Brown LR, Campargue A, Chance K, Cohen EA, Coudert LH, Devi VM, Drouin BJ, Fayt A, Flaud J-M, Gamache RR, Harrison JJ, Hartmann J-M, Hill C, Hodges JT, Jacquemart D, Jolly A, Lamouroux J, Le Roy RJ, Li G, Long DA, Lyulin OM, Mackie CJ, Massie ST, Mikhailenko S, Müller HSP, Naumenko OV, Nikitin AV, Orphal J, Perevalov V, Perrin A, Polovtseva ER, Richard C, Smith MAH, Starikova E, Sung K, Tashkun S, Tennyson J, Toon GC, Tyuterev VG, Wagner G. The HITRAN 2012 molecular spectroscopic database. J Quant Spectrosc Radiat Transf. 2013;130:4-50.

32. Gu C, Zuo Z, Luo D, Peng D, Di Y, Zou X, Yang L, Li W. High-repetition-rate femtosecond mid-infrared pulses generated by nonlinear optical modulation of CW QCLs and ICLs. Opt Lett. 2019;44:5848-51.

\section{Publisher's Note}

Springer Nature remains neutral with regard to jurisdictional claims in published maps and institutional affiliations.

\section{Submit your manuscript to a SpringerOpen ${ }^{\circ}$ journal and benefit from:}

- Convenient online submission

- Rigorous peer review

- Open access: articles freely available online

- High visibility within the field

- Retaining the copyright to your article

Submit your next manuscript at $>$ springeropen.com 